\title{
Der Berg Garizim im Deuteronomium
}

\author{
Detlef Jericke \\ (Universität Heidelberg; detlef.jericke@wts.uni-heidelberg.de)
}

\section{Einleitung}

Die Ortsangabe »Berg Garizim « findet sich im Deuteronomium in den Kapiteln 11 und 27 jeweils an den Nahtstellen zwischen den Rahmenkapiteln (Dtn 1-11 und Dtn 27-34) und dem literarischen Zentrum des Buchs (Dtn 12-26). Allein aufgrund dieser exponierten Stellung1 erscheint eine Untersuchung des Toponyms angebracht. Zugleich sind mit dem Ortsnamen religionsgeschichtliche Fragen verknüpft. Am Garizim bildete sich in der zweiten Hälfte des 1. Jts. v. Chr. eine eigenständige Kultgemeinde, die ebenso wie die Gemeinde am Tempel in Jerusalem einen regulären Jhwh-Kult für sich beanspruchte. ${ }^{2}$ Noch vor wenigen Jahren schienen die Anfänge der Gemeinde auf dem Garizim lediglich in Umrissen fassbar. Da keine archäologischen Reste aus vorhellenistischer Zeit bekannt waren, ${ }^{3}$ folgerte man aus den Schilderungen bei Josephus (Antiquitates 11, 302-347; 13, $254 \mathrm{ff}$.), dass die definitive Trennung von der Gemeinde in Jerusalem erst in der Makkabäerzeit ${ }^{4}$ oder in römischer

1 Vgl. M. Geiger, Gottesräume. Die literarische und theologische Konzeption von Raum im Deuteronomium, BWANT 183, 2010, 256.308.

2 M. Kartveit, The Origin of the Samaritans, VT.S 128, 2009; M. Mor/F. V. Reiterer (Hg.), Samaritans: Past and Present. Current Studies, SJ 53 = Studia Samaritana 5, 2010.

3 Aus älteren Grabungen waren lediglich die Reste der oktogonalen Theotokos-Kirche aus dem 5. Jh. n. Chr. auf dem Gipfelplateau und ein kleiner Zeus/Jupiter-Tempel aus spätrömischer Zeit (2.-4. Jh. n. Chr.) auf den Tell er-Rās (Palestine Grid 1762.1793), einem dem Garizim vorgelagerten Bergsporn, bekannt; vgl. I. Magen, Art. Gerizim, Mount, in: E. Stern u.a. (Hg.), NEAEHL 2, 1993, 484-492; I. Magen, Mount Gerizim and the Samaritans, in: F. Manns/E. Alliata (Hg.), Early Christianity in Context. Monuments and Documents, SBF.CMa 38, 1993, 91-148, 122-127. Vom Talgrund führten in der Antike Stufen hinauf zum Tell er-Rās. In Teilen sind sie archäologisch nachgewiesen. Zudem sind Stufen und Tempel auf römischen Münzen abgebildet: Magen, Mount Gerizim, 122-127; J. Zangenberg, Berg des Segens, Berg des Streits. Heiden, Juden, Christen und Samaritaner auf dem Garizim, ThZ 63/4 (2007), 289-309, 306 Abb. 4.

4 H. G. Kippenberg, Garizim und Synagoge. Traditionsgeschichtliche Untersuchungen zur samaritanischen Religion der aramäischen Periode, 1971, 60-93. 
Zeit erfolgte. ${ }^{5}$ Somit bestand kein Anlass, die alttestamentlichen Garizim-Belege mit der vermeintlich späteren samaritanischen Kultgemeinde in Verbindung zu bringen. Inzwischen haben sich die Voraussetzungen geändert. Neuere Ausgrabungen legten auf dem Gipfelplateau des Garizim unter der Theotokos-Kirche ${ }^{6}$ eine ca. 40 ha große Siedlung frei. Sie stammt aus hellenistischer Zeit (2. Jh. v. Chr.). Im Zentrum befand sich ein ca. $150 \times 100$ m großer ummauerter Bezirk, der als Kultbezirk gedeutet wird. Ältere Mauerzüge unter den Umfassungsmauern der hellenistischen Zeit werden von den Ausgräbern in persische Zeit (5./4. Jh. v. Chr.) datiert. Dieser Befund deutet darauf hin, dass das erste Heiligtum der Gemeinde auf dem Garizim bereits in persischer Zeit als Gegenstück zum Jerusalemer Tempel errichtet wurde. ${ }^{7}$ Gleichzeitig hat sich die Deuteronomiumsforschung von der Prämisse gelöst, das Buch sei weitgehend in der israelitisch-judäischen Königszeit entstanden. ${ }^{8}$ Vielmehr wird die literarische Entstehungsgeschichte bis in die persische Zeit hinein ausgezogen. ${ }^{9}$ Archäologisch bezeugte Anfänge des Kults auf dem Garizim und Entstehungszeit des Deuteronomiums sind demnach in zeitliche Nähe zueinander gerückt. Damit eröffnet sich die Möglichkeit, die entsprechenden Belege zum Garizim im Hinblick auf ihre religionsgeschichtliche Aussagekraft erneut zu untersuchen.

\section{Deuteronomium 11}

Dtn 11,29 bringt die Anweisung, Israel solle nach der Ankunft im Land »den Segen auf dem Berg Garizim und den Fluch auf dem Berg Ebal geben «. Der darauf folgende Vers Dtn 11,30 beschreibt die Lage der beiden Berge in einer als Frage formulierten, syntaktisch komplexen Fügung:

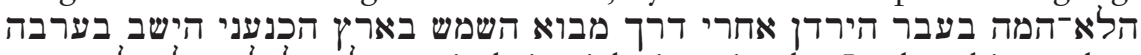
sind sie nicht jenseits des Jordan, hinter dem Weg zum Sonnenuntergang, im Land des Kanaaniters, der in der Araba

5 M. Sasse, Geschichte Israels in der Zeit des Zweiten Tempels. Historische Ereignisse Archäologie - Sozialgeschichte - Religions- und Geistesgeschichte, 2004, 85-92.

6 A.M. Schneider, Römische und byzantinische Bauten auf dem Garizim, ZDPV 68 (1951), 211-234.

7 I. Magen, Gerizim, Mount, in: E. Stern u.a. (Hg.), The New Encyclopedia of Archaeological Excavations in the Holy Land. Supplementary Volume, 2008, 1742-1748; I. Magen/H. Misgav/L. Tsfania, Mount Gerizim Excavations. 2. A Temple City, Judea \& Samaria Publications 8, 2008; I. Hjelm, Mt. Gerizim and Samaritans in Recent Research, in: Mor/Reiterer (Hg.), Samaritans, 25-41.

8 So noch G. von Rad, Das fünfte Buch Mose. Deuteronomium, ATD 8, 3. Aufl. 1978; J.H. Tigay, Deuteronomy, דברים, The JPS Torah Commentary, 1996.

9 J.C. Gertz, Die Literatur des Alten Testaments. I. Tora und Vordere Propheten, in: Ders. (Hg.), Grundinformation Altes Testament. Eine Einführung in Literatur, Religion und Geschichte des Alten Testaments, 3. Aufl. 2009, 193-311, 247-260. 
wohnt, gegenüber Gilgal neben den großen Bäumen More? «10. Erklärungsbedürftig ist das Nebeneinander von Ortsangaben, die in den Jordangraben weisen (Araba, Gilgal) ${ }^{11}$ und solchen, die aus der Gegend von Sichem im zentralpalästinischen Bergland bekannt sind (More, vgl. Gen 12,6f.). Der samaritanische Pentateuch (Smr) verschärft die Fragestellung, indem er More ausdrücklich bei Sichem lokalisiert (אלון מורא מול שכם) und somit klarstellt, dass es sich um den aus der Abrahamgeschichte bekannten Platz handelt.

Meist werden die beiden lokalen Näherbestimmungen in Dtn 11,30 so gedeutet, dass die erstgenannte "falsch, die andere richtig " 12 ist. Die Lokalisierung des Garizim bei Sichem ist allerdings keineswegs eindeutig. Lediglich Jdc 9,7 setzt eine solche Lage voraus. Die Belegstellen im Deuteronomium und im Josuabuch lassen die Lokalisierung entweder offen (Dtn 27,4[Smr].12) oder bringen neben den bereits erwähnten Ortsangaben im Jordangraben (Dtn 11) die Gegend von Ai (et-Tell, Palestine Grid 1748.1471) im palästinischen Bergland nördlich von Jerusalem ins Spiel (Jos 8,33). Die Septuaginta (LXX) allerdings stellt Jos 8,30-35 hinter Jos $9,1 \mathrm{f}$. und bringt Garizim und Ebal so näher an Sichem heran, indem die beiden Berge erst nach der Einnahme von Ai und nach dem Stichwort "Gebirge " in Jos 9,1 genannt werden. ${ }^{13}$ Auf diese Weise verdeutlicht LXX, dass Garizim und Ebal zum Jos 9,1 genannten »Gebirge « gehören.

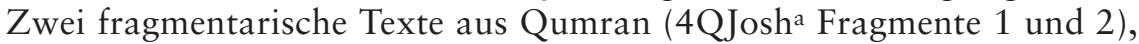
die einen erweiterten Text von Jos 8,34f. und den Anfang von Jos 5,2 enthalten, lassen sich dagegen in der Weise deuten, dass die dortigen Schreiber Jos 8,30-35 zwischen Jos 4 und 5 stellten, um auf diese Weise den $\mathrm{Zu}$ sammenhang von Garizim und Ebal mit Ai zu vermeiden und den Text mit den geographischen Vorstellungen von Dtn 11 auszugleichen. ${ }^{14}$ Noch Eusebius lokalisiert Ebal und Garizim bei Jericho, also in der Nähe von Gilgal. Allerdings kennt er bereits eine samaritanische Tradition, die den Garizim bei Sichem verortet. Diese Tradition hält er jedoch für einen Irr-

10 Zur Übersetzung des Wortes אלוני s. D. Jericke, Abraham in Mamre. Historische und exegetische Studien zur Region von Hebron und zu Genesis 11,27-19,38, CHANE 17, 2003, $35 \mathrm{f}$.

11 Die alttestamentliche Überlieferung lässt keinen Zweifel, dass Gilgal im Jordantal bei Jericho zu suchen ist (Jos 4,19; 5,10). Auch die Mosaikkarte von Medeba verzeichnet den Platz hier (H. Donner, The Mosaic Map of Madaba. An Introductory Guide, 1992, 46, Nr. 26). Zu den Lokalisierungsvorschlägen vgl. K. Bieberstein, Art. Gilgal, RGG4, Bd. 3, 2000, 930-931.

12 E. Nielsen, Deuteronomium, HK I/6, 1995, 130.

13 Vgl. C. Nihan, The Torah between Samaria and Judah: Shechem and Gerizim in Deuteronomy and Joshua, in: G. N. Knoppers/B. M. Levinson (Hg.), The Pentateuch as Torah. New Models for Understanding Its Promulgation and Acceptance, 2007, 187-223, 219.

14 Nihan, The Torah, $220 \mathrm{f}$. 
tum (Onomastikon 64,9-17). ${ }^{15}$ Die Mosaikkarte von Medeba nimmt die Erörterungen von Eusebius maximalistisch auf, indem sie den Berg Garizim zweimal darstellt, einmal unter dem Namen ГAPIZEIN östlich von Gilgal und Jericho, ein zweites Mal als TOYP ГAPIZIN westlich von Sichem bzw. südlich von Neapolis, der römischen Nachfolgesiedlung von Sichem. ${ }^{16}$ Aufgrund der seit der Antike belegten samaritanischen Lokaltraditionen und der neueren Ausgrabungsfunde gilt heute die Lokalisierung der beiden Berge bei der Stadt Nāblus, also im Bereich von Neapolis bzw. Sichem als gesichert. Der Garizim wird mit dem südwestlich der Stadt gelegenen Gebel eț-Tōor (Palestine Grid 1759.1785), der Berg Ebal mit dem Ğebel Islāmìye (Palestine Grid 1760.1820) nördlich von Nāblus gleichgesetzt. ${ }^{17}$

Insofern bleibt in Dtn 11,30 allein die lokale Näherbestimmung der beiden Berge durch Ortsangaben, die in den Jordangraben weisen, erklärungsbedürftig. Da eine historisch-topographische Lösung ausgeschlossen ist, sollte nach einer literarisch-topographischen Erklärung gesucht werden. Veijola deutet eine solche an, wenn er ausführt, dass »Geographie hier im Dienste theologischer Polemik steht «. ${ }^{18}$ In V. 29 erkennt er das Bemühen, »im Namen einer nationalen Versöhnung den kultischen Ansprüchen der Samaritaner ein gewisses Recht einzuräumen «. ${ }^{19}$ Diese Tendenz werde in V. 30 »durch topographische Mittel zunichte " gemacht, indem ein »den samaritanischen Ansprüchen feindlich gesinnter Glossator « versuche, »deren heiligste Tradition dadurch zu falsifizieren,

15 R. S. Notley, Z. Safrai, Eusebius, Onomasticon. The Place Names of Divine Scripture. Including the Latin Edition of Jerome Translated into English and with Topographical Commentary, 2005, 63 f., Nr. $307 \mathrm{f}$.

16 Donner, Mosaic Map, 48. Kartveit, Origin, 234, macht darauf aufmerksam, dass die Namensform ГAPIZEIN der LXX-Schreibung folgt, während TOYP ГAPIZIN eine Transliteration des von den Samaritanern verwendeten aramäischen Namens ist.

17 Am nordöstlichen Berghang (Palestine Grid 1775.1824) wurde eine ca. $250 \times 52 \mathrm{~m}$ große ummauerte Anlage der Eisenzeit I (12./11. Jh. v. Chr.) mit einer ca. $9 \times 7$ m großen Installation aus unbehauenen Steinen ausgegraben. Der Ausgräber deutet die Installation als Altar und bringt sie mit Jos 8,30-35 in Verbindung (A. Zertal, An Early Iron Age Cultic Site on Mount Ebal: Excavation Seasons 1982-1987, Tel Aviv 13-14 [1986-1987], 105-189; Ders., Art. Ebal, Mount, in: E. Stern, u.a. [Hg.], NEAEHL 1, 1993, 375-377). Ähnliche Bauwerke der Eisenzeit I aus dem Negeb und aus Giloh (Palestine Grid 1678.1265) südlich von Jerusalem werden als landwirtschaftliche Siedlungen angesehen (A. Mazar, Giloh: An Early Israelite Settlement Site Near Jerusalem, IEJ 31 [1981], 1-36). Diese Interpretation dürfte auch auf die Funde vom Ebal zutreffen (A. Kempinski, Joshua's Altar - An Iron Age I Watchtower, BArR 12/1 [1986], 42-49). Kultische Aktivitäten sind daher lediglich auf dem Garizim ab der Mitte des 1. Jts. v. Chr., nicht jedoch auf dem Ebal archäologisch nachzuweisen.

18 T. Veijola, Das 5. Buch Mose Deuteronomium. Kapitel 1,1-16,7, ATD 8,1, 2004, 259.

19 Veijola, Deuteronomium, 258. 
dass er ihre Berge Garizim und Ebal in die Gegend des Jordanübergangs verlegt «. Gleichzeitig bleibt es für Veijola jedoch »dunkel «, warum derselbe Glossator »es für nötig hält, auch die aus der Nähe von Sichem bekannte >Orakelterebinthe (Gen 12,6) hierher umzupflanzen «. ${ }^{20}$ Der Versuch, literarkritische Beobachtungen mit Gesichtspunkten der literarischen Topographie zu verbinden, bringt demnach Veijola nicht zu einer befriedigenden Lösung. Darüber hinaus zeigt die erwähnte Texterweiterung des Smr in Dtn 11,30 mit dem Hinweis auf die Lage von More bei Sichem, dass die Samaritaner den Vers konstruktiv aufgriffen und ihn keineswegs als ihnen feindlich gesinnte »Glosse "verstanden.

Eine literarisch-topographische Erklärung bietet sich demgegenüber an, wenn der Erzählzusammenhang ab Dtn 9 einbezogen wird. Dtn 9,1 lenkt den Blick auf die aktuelle Situation der Israeliten (»heute «) vor dem Übergang über den Jordan und dem Einzug in das von Jhwh zugesagte Land, dessen Inbesitznahme Israel jedoch aus eigener Kraft nicht leisten kann. ${ }^{21}$ Jhwh streitet für Israel, obwohl das Volk während der Zeit der Wüstenwanderung mehrfach gegen ihn rebelliert hat. Der alleinige Grund für Jhwhs Eingreifen liegt in der Einlösung der an Israels »Väter « Abraham, Isaak und Jakob ergangenen Zusagen (Dtn 9,4-6; vgl. Dtn 10,11.15; 11,9.21). Dtn 9,7-11,32 entfalten die Thematik von Dtn 9,1-6 in drei Schritten:22

(1) Zunächst ist Israel im Blick (Dtn 9,7-11,1). Im Rückgriff auf die Wüstenwanderungszeit wird an die Auflehnung Israels gegen Jhwh erinnert (Dtn 9,7-10,11). Insbesondere die Ereignisse »in Horeb « (Dtn 9,8) mit der Errichtung des Stierbilds illustrieren den Ungehorsam der Israeliten. Dabei wird mehrfach zwischen Dtn 9,9 und Dtn 10,10 die Ortsangabe »Berg " wiederholt. ${ }^{23}$ Anschließend wird nochmals eingeschärft, dass Israel künftig die Weisungen Jhwhs befolgen soll (Dtn $10,12-11,1) .{ }^{24}$

(2) Der zweite Argumentationsgang gilt Jhwh (Dtn 11,2-25).25 Ana$\log$ zum Aufbau des ersten Erzählschritts wird zunächst auf seine bereits

20 Beide Zitate Veijola, Deuteronomium, 259.

21 Zu Dtn 9,1 als erzählerischem Neueinsatz vgl. N. Lohfink, Deuteronomium 9,1-10,11 und Exodus 32-34. Zu Endtextstruktur, Intertextualität, Schichtung und Abhängigkeiten, in: M. Köckert/E. Blum (Hg.), Gottes Volk am Sinai. Untersuchungen zu Ex 32-34 und Dtn 9-10, VWGTh18, 2001, 41-87.

$22 \mathrm{Zu} \mathrm{Dtn} \mathrm{9,1-6}$ als »Einleitung in Moses dritte Horeberzählung $(9,7-10,11)$ «s. Geiger, Gottesräume, 198.

23 »Auf dem Berg « בהר (Dtn 9,9f.; 10,4.10); »auf den Berg «הרה (Dtn 9,9; 10,1.3); »vom Berg "מן־ההר (Dtn 9,15.21;10,5).

24 Der Neueinsatz in Dtn 10,12 wird wie in Dtn 9,1 durch die direkte Anrede an Israel markiert; vgl. Lohfink, Deuteronomium 9,1-10,11, 42; Geiger, Gottesräume, 225.

25 Wie in Dtn 9,1 setzt der Abschnitt mit der Zeitangabe »heute« ein. 
geschehene Rettungstat verwiesen (Dtn 11,3-5) und anschließend der Blick in die Zukunft gerichtet: Jhwh bringt Israel in ein Land, das er vorzüglich ausstattet (Dtn 11,8-15) und das bis an die Grenze des Zweistromlands reicht (Dtn 11,24).

(3) Der gesamte Erzählbogen wird abgeschlossen durch die Beschreibung des ersten Akts in dem Land, das durch Überschreiten des Jordans (Dtn 9,1) betreten wird (Dtn 11,26-32):26 einer Segen- und Fluchzeremonie, bei der die Handlungen Jhwhs und Israels eng aufeinander bezogen sind. Bereits "heute", also vor dem Jordanübergang, wird Jhwh Segen und Fluch vor Israel "geben" (Dtn 11,26). In Entsprechung dazu soll Israel unmittelbar nach dem Einzug in das Land Segen und Fluch auf den Bergen Garizim und Ebal "geben " (Dtn 11,29). Die Entsprechung zwischen der Vorgabe Jhwhs (V. 26) und der Befolgung durch Israel (V. 29) הברכה) הird zusätzlich durch die Determinierung der Nomina in V. 29 bzw. הקללה) ausgedrückt: es handelt sich um eben den Segen und den Fluch, den Jhwh »heute " bereits vorgibt (V. 26). Dtn 11,31f. lenken den Blick mit dem Hinweis auf den Jordanübergang auf den Beginn des gesamten Erzählbogens in Dtn 9,1 zurück und schließen mit der wiederholten Aufforderung, Jhwhs Weisungen zu halten als Voraussetzung dafür, dauerhaft und sicher im Land zu wohnen.

Im Erzählzusammenhang von Dtn 9-11 werden die Ortsangaben Garizim und Ebal demnach mit der ersten Aktion Israels im Land nach dem Jordanübergang verbunden. Die beiden Berge werden zu Orten einer Anfangshandlung, einem für das dauerhafte Wohnen im Land entscheidenden Nachweis des Gehorsams gegen Jhwhs Vorgaben.27 Im Josuabuch, bei der erzählerischen Ausführung der Landnahme, erfolgt die erste Aktion nach dem Jordanübergang dagegen in Gilgal. Dort stellt Josua die zwölf aus dem Jordan mitgenommenen Steine auf (Jos 4,19f.). In Gilgal wird auch das erste Pesach im Land Kanaan gefeiert (Jos 5,9f.). Indem Dtn 11,30 Garizim und Ebal mit der Ortsangabe Gilgal verbindet, werden literarisch die jeweiligen Orte einer Anfangshandlung im Land zusammengebracht. ${ }^{28}$ Dadurch werden Garizim und Ebal in ihrer Bedeutung nicht entwertet, wie Veijola meint, sondern aufgewertet. Die zweite lokale Näherbestimmung der Berge in Dtn 11,30 durch die Ortsangabe "More" verweist auf den Altarbau Abrahams (Gen 12,6f.). Auch hier handelt es sich um eine Anfangshandlung, um die erste Aktion Abrahams nach der Ankunft im Land Kanaan. Der Rückbezug auf Abraham ist umso wichtiger, als die an die »Väter« ergangene Landverheißung die

\footnotetext{
26 V. 26 zeigt Entsprechungen zu Dtn 9,1a sowohl in dem Aufmerksamkeitsruf am Beginn des Verses als auch in der Zeitangabe "heute".

27 Vgl. Tigay, Deuteronomy, 486; Geiger, Gottesräume, 254.

28 Geiger, Gottesräume, 249-251.
} 
alleinige Grundlage für die Landgabe an Israel bildet (Dtn 9,4-6). Dtn 11,30 verbindet somit drei Ortsangaben, die auf Anfangshandlungen im Land Kanaan verweisen.

\section{Deuteronomium 27}

Dtn 27 nimmt die in Dtn 11,29f. mit verschiedenen Ortsangaben verbundenen Motive auf: Segen und Fluch (Garizim und Ebal), Aufrichtung von Steinen (Gilgal) und Altarbau (More). Dabei werden die jeweiligen kultischen Anfangshandlungen literarisch-topographisch zusammengeführt und alle drei an den Bergen Ebal und Garizim lokalisiert. Auf dem Ebal (masoretischer Text $=\mathrm{M}$ ) bzw. auf dem Garizim (Smr) sollen Steine aufgestellt werden (Dtn 27,4, vgl. V. 2f.), auf die "alle Worte dieser Weisung" geschrieben werden $(27,3.8)$. Gleichzeitig soll dort ein Altar aus unbehauenen Steinen errichtet werden (27,5-7). Die in Dtn 11,29 mit dem Terminus "geben " umschriebene Zeremonie auf den beiden Bergen wird dahingehend erläutert, dass sich jeweils sechs Stämme auf dem Garizim $(27,12)$ und auf dem Ebal $(27,13)$ aufstellen sollen, um das Volk zu segnen bzw. zu verfluchen.

Die topographische Engführung der drei Anfangshandlungen unterstreicht den Stellenwert von Garizim und Ebal innerhalb der literarischtopographischen Inszenierung des Deuteronomiums. Dabei sind Unterschiede zwischen der masoretischen und der samaritanischen Überlieferung erkennbar. Diese lassen sich zunächst in Dtn 27,4f. mit der Ortsangabe, die den Platz für die Aufrichtung der Steine (V. 4) und des Altars (V. 5) kennzeichnet, festmachen. M liest in V. 4 »auf dem Berg Ebal « בהר עיבל Dieser Version folgt die Hauptüberlieferung der LXX. Smr dagegen schreibt "auf dem Berg Garizim " בהרגר(י)וים. Der griechische Text des Papyrus Giessen 19 bietet mit $\alpha \rho \gamma \alpha \rho(1) \zeta ı \mu$ eine auf der Lesart des Smr basierende Transkription. ${ }^{29}$ Noch bis vor wenigen Jahren folgten die Auslegungen zumeist der masoretischen Textform und erklärten die Variante "auf dem Berg Garizim» als eine von der samaritanischen Gemeinde vorgenommene Korrektur zur Legitimierung ihres Hei-

29 Vgl. BHQ 5, 122\%f.; A. Schenker, Textgeschichtliches zum Samaritanischen Pentateuch und Samareitikon. Zur Textgeschichte des Pentateuchs im 2. Jh. v. Chr., in: Mor/Reiterer (Hg.), Samaritans, 105-121, 108-113. Auch ein im Jahr 2009 von der Azusa Pacific University vorgestelltes, im Antikenhandel erworbenes Textfragment, das nach ersten Angaben aus Qumran stammen soll und vorläufig in das 2./1. Jh. v. Chr. datiert wird, zeigt einen mit Smr identischen Text: S. Kreuzer, Vom Garizim zum Ebal. Erwägungen zur Geschichte und Textgeschichte sowie zu einem neuen Qumran-Text, in: U. Dahmen/ J. Schnocks (Hg.), Juda und Jerusalem in der Seleukidenzeit. Herrschaft - Widerstand Identität (Festschrift Fabry), BBB 159, 2010, 31-42, mit Hinweisen auf die entsprechenden Internetseiten. 
ligtums. ${ }^{30}$ Neuere Studien dagegen halten die Lesart des Smr für ursprünglich und die Ortsangabe des $M$ für eine spätere Korrektur. ${ }^{31} \mathrm{Ni}$ han stützt mit der entsprechenden textkritischen Entscheidung die These, dass die Pentateuchredaktion, die er in persische Zeit datiert, sowohl die Interessen der Jerusalemer Gemeinde als auch diejenigen der neu entstandenen Kultgemeinschaft auf dem Garizim berücksichtigte. Daher sei der Garizim im Deuteronomium ausdrücklich als Ort des Segens genannt. ${ }^{32}$

Eine wichtige Rolle bei der Frage der textkritischen Beurteilung von Dtn 27,4 spielt Dtn 27,13, wo der Berg Ebal als der Ort genannt ist, von dem aus sechs Stämme den Fluch sprechen sollen. Weil der Ebal für den Fluch vorgesehen ist, so die Argumentation von Nihan, könne er nicht ursprünglich auch als Platz der Aufrichtung der Steine und des Altars genannt gewesen sein. ${ }^{33}$ Umgekehrt meint Tigay, wenn ein judäischer Bearbeiter ein ursprüngliches »Garizim « nachträglich in "Ebal « geändert hätte, dann hätte er auch in Dtn 27,12f. die Ortsangaben vertauscht und den Platz des Segens »Ebal «, den Platz des Fluches »Garizim « genannt. ${ }^{34}$ Differenzierter argumentiert Michaela Geiger. ${ }^{35}$ Auch sie hält die Lesart »Garizim « in Dtn 27,4 für ursprünglich. Allerdings versteht sie den Text nicht als einen Versuch, die Interessen der samaritanischen Gemeinde zu berücksichtigen. Der Garizim sei als Ort einer »Eingangsliturgie « 36 nach dem Betreten des Landes Kanaan gewählt, weil er als Berg »die Qualität des HOREB « repräsentiere, ${ }^{37}$ der wiederum in der »Raumkonzeption « des Deuteronomiums eine zentrale Stelle einnehme. ${ }^{38}$ Gleichzeitig geht

30 Nielsen, Deuteronomium 1995, 246; Tigay, Deuteronomy, 394 Anm. 12; E. Noort, The Traditions of Ebal and Gerizim. Theological Positions in the Book of Joshua, in: M. Vervenne/J. Lust (Hg.), Deuteronomy and Deuteronomistic Literature (Festschrift Brekelmans), BEThL 133, 1997, 161-180, 166-168; S.L. Richter, The Place of the Name in Deuteronomy, VT 57 (2007), 342-366, 347.

31 U. Rüterswörden, Das Buch Deuteronomium, NSK.AT 4, 2006, 172 f.; Nihan, The Torah, 213 f.; Kartveit, Origin, 300-309; Kreuzer, Vom Garizim zum Ebal, 35-37; Schenker, Textgeschichtliches, 106-113; Geiger, Gottesräume, 252; in der Tendenz auch BHQ 5, $122 *$ f.; vgl. schon von Rad, Deuteronomium, $117 \mathrm{f}$. In der ersten Auflage des Kommentars, die 1964 erschien, findet sich noch die Diskrepanz zwischen Übersetzung (»Ebal «) und Kommentar, der die Lesart » Garizim « voraussetzt.

32 Nihan, The Torah, $213 \mathrm{f}$.

33 Nihan, The Torah, 213.

34 Tigay, Deuteronomy, 394 Anm. 12.

35 Geiger, Gottesräume, 251-256.

36 Geiger, Gottesräume, 254.

37 Geiger, Gottesräume, 299.

38 »Gravitationszentrum des Dtn ist jedoch der Horeb, der im Dtn als Ursprung Israels inszeniert wird ..." (Geiger, Gottesräume, 70). Geiger versteht das Toponym »Horeb « im Deuteronomium durchgängig als Name eines Berges. Der Textbefund deutet jedoch darauf hin, dass »Horeb« im Deuteronomium eine Region meint, vgl. L. Perlitt, Sinai und 
Geiger davon aus, dass die Einrichtung des Kults auf dem Garizim die Textänderung in »Ebal « veranlasst hat: »Mit der Ersetzung des Garizim durch den Ebal versucht der MT, der Dauerhaftigkeit des dortigen, samaritanischen Kultes die Grundlage zu entziehen - um den Preis allerdings, dass das erste im Land gefeierte Ritual mit dem Fluchberg verbunden wird «. ${ }^{39}$ Die unterschiedlichen Auslegungen von Nihan und Geiger deuten somit an, dass bei der Frage der Textkritik in Dtn 27,4 auch überlieferungsgeschichtliche und v.a. religionsgeschichtliche Überlegungen miteinbezogen werden sollten.

Ein weiteres Problem betrifft Jos 8,30-35, wo die Ausführung der in Dtn 11,29f. und Dtn 27,2-13 angewiesenen Maßnahmen erzählt wird. Der Altarbau und die Beschriftung der Steine (Jos 8,30-32) finden »auf dem Berg Ebal « statt (Jos 8,30) und setzen damit die Version von M in Dtn 27,4 voraus. Wer in Dtn 27,4 dem Smr folgt und ein ursprüngliches »Garizim « postuliert, muss entweder eine solche Lesung auch für Jos 8,30 voraussetzen, zwangsläufig ohne erkennbaren Anhalt in der Textüberlieferung, oder die Entstehung von Jos 8,30-35 in eine Zeit verlegen, als die »judäische " bzw. antisamaritanische Korrektur in Dtn 27,4 bereits erfolgt und damit der Ebal als Ort des Geschehens festgeschrieben war. ${ }^{40}$

\section{Deuteronomium 12}

Aussagekräftig für die Frage nach den textkritischen Verhältnissen in Dtn 27,4 sind auch die Unterschiede von M und Smr in Dtn 12. Während M die Entscheidung für den Platz, an dem Jhwh seinen Namen setzen will, in die Zukunft verlegt und deshalb an den entsprechenden Textstellen (Dtn 12,5.11.14.18.21.26) die Präformativform יבחר "er wird auswählen« hat, findet sich in Smr hier durchgängig die Affirmativform בחר קer

Horeb, in: H. Donner u.a. (Hg.), Beiträge zur Alttestamentlichen Theologie (Festschrift Zimmerli), 1977, 302-322, 318f. = L. Perlitt, Deuteronomium-Studien, FAT 8, 1994, 32-49, 45 f.

39 Geiger, Gottesräume, 254f. Der Ebal als Ort des Fluchs ist allerdings nicht zwangsläufig negativ konnotiert. In altorientalischen Verträgen spielen Flüche eine konstitutive Rolle. Sollte das Deuteronomium teilweise nach dem Muster altorientalischer Vasallenverträge oder Treueide gestaltet sein, so müssten Segen und Fluch differenzierter bewertet werden; vgl. C. Koch, Vertrag, Treueid und Bund. Studien zur Rezeption des altorientalischen Vertragsrechts im Deuteronomium und zur Ausbildung der Bundestheologie im Alten Testament, BZAW 383, 2008.

40 Nihan, The Torah, 222, bringt die Einführung der Lesart »Ebal « in Dtn 27 mit den Auseinandersetzungen zwischen den Hasmonäern und der Gemeinde am Garizim, die in der Kampagne von Johannes Hyrkan 112/111 v.Chr. kulminierten, in Zusammenhang. Dementsprechend beschreibt er Jos 8,30-35 als "postcanonical revision «, was bedeutet, dass der Text an der Schwelle zum 1. Jh. v. Chr. entstand. Rüterswörden, Deuteronomium, 173, begnügt sich mit dem Hinweis, Jos 8,30-35 sei ein später Zusatz. 
hat ausgewählt « ${ }^{41}$ Nach samaritanischer Tradition hat Jhwh den Platz, an dem er kultisch verehrt werden will, bereits gewählt. Da Sichem der erste Platz in Kanaan ist, an dem sich Jhwh zeigt (Gen 12,6f.), und da Sichem in Dtn 11,29f. im Zusammenhang mit Garizim und Ebal nochmals erwähnt wird, ist die Schlussfolgerung zwingend, dass für die samaritanische Tradition der Garizim die in Dtn 12 umschriebene legitime Kultstätte Jhwhs ist.

Die weitergehende Frage ist nun, ob textkritische Entscheidungen für den Einzelfall getroffen werden oder ob bestimmten Textformen durchgehend Priorität eingeräumt wird. Die Mehrheit der Auslegenden wählt den ersten Lösungsweg. So folgen etwa von Rad und Rüterswörden in Dtn 12 dem masoretischen Text, ${ }^{42}$ obgleich sie sich in Dtn 27,4 für die samaritanische Lesart entscheiden. Dementsprechend relativieren sie die mögliche Verbindung von Dtn 27,4 zu Dtn 12. Die in diesem Zusammenhang vorgebrachten Begründungen sind schwer nachvollziehbar ${ }^{43}$ oder durch neuere Forschungen überholt. ${ }^{44}$ Nihan erwähnt die textkritischen Alternativen in Dtn 12 anmerkungsweise, lässt aber eine Entscheidung für die eine oder andere Variante offen. ${ }^{45}$ Für Schenker hingegen repräsentieren die Lesungen des Smr sowohl in Dtn 27,4 als auch in Dtn 12 die ursprünglichere Textform. Der Text von $\mathrm{M}$ gehe in beiden Fällen auf Korrekturen im "proto-massoretischen Text « zurück. ${ }^{46}$ Schenker führt zur Begründung an, dass Smr in Dtn 27,4 und Dtn 12 den Wortlaut der "ursprünglichen Septuaginta " bewahrt hat. Die Argumentation ist vergleichsweise kompliziert, zumal es sich bei der »ursprünglichen Septuaginta « um eine lediglich erschlossene Größe handelt. Zu Dtn 27,4 verweist Schenker einmal auf eine Handschrift der Vetus Latina, den Lyoner Heptateuch (»Lugdunensis«), der wie Smr»Garizm» liest. Da die Vetus Latina die lateinische Übersetzung der Septuaginta sei und im vorliegenden Fall Dtn 27,4 nicht mit Jos 8,30 ausgleiche, entspreche sie »der ur-

41 Vgl. BHQ 5, 84*f.

42 So auch Veijola, Deuteronomium, 267.

43 Rüterswörden interpretiert den nach Dtn 27,4f. (Smr) auf dem Garizim aufzustellenden Altar als »Interimslösung eines Jahwealtars, der zwar nicht zentralisiert, gleichwohl legitim ist. Bei der Zentralisation sollen diese Kultstätten nach Dtn 12,4 nicht zerstört, sondern aufgelassen werden « (Rüterswörden, Deuteronomium, 172).

44 Von Rad begründet seine Zweifel mit dem Hinweis, aus der Königszeit, deren Verhältnisse das Deuteronomium voraussetze, seien keine kultischen Aktivitäten auf dem Berg Garizim bekannt (von Rad, Deuteronomium, 118). Da neuere archäologische und exegetische Erkenntnisse dafür sprechen, die literarische Entstehung des Deuteronomiums und die archäologisch nachgewiesenen Anfänge des Kultbetriebs auf dem Garizim in zeitliche Nähe zueinander zu rücken (s.o.), fallen diese Vorbehalte weg.

45 Nihan, The Torah, 216.

46 Schenker, Textgeschichtliches, 116. 
sprünglichen Lesart der Septuaginta «. ${ }^{47}$ Weiterhin argumentiert Schenker mit dem Papyrus Giessen 19. Da dieser die in der samaritanischen Textüberlieferung gebräuchliche Namensform transkribiere (s.o.), sei als Verfasser ein Samaritaner vorauszusetzen. Der Text entspreche daher vielleicht dem nicht erhaltenen Samareitikon, der griechischen Übersetzung des Smr. ${ }^{48}$ Im Falle von Dtn 12 führt Schenker aus, dass an einigen Textstellen, in denen Smr die Affirmativform hat, LXX-Varianten dieser Lesung folgen. Daraus schließt er: »Die Lesarten mit dem Verb in der Vergangenheit in der deuteronomischen Formel von Gottes Erwählung des heiligen Ortes entspricht [sic] nach alledem der ursprünglichen Septuaginta ${ }^{49} \mathrm{Da}$ »ursprüngliche Septuaginta « und Smr zusammen gehen, sei es »höchst wahrscheinlich, dass sie die ursprüngliche Lesart bewahren «50. Aus den textkritischen Entscheidungen zieht Schenker eher vorsichtige religions- und literaturgeschichtliche Schlussfolgerungen. Er gesteht zu, dass Verse wie Dtn 11,30 (Smr), Dtn 27,4 (Smr) oder Jos 8,33 die Interpretation nahe legen konnten, »die von Gott gewählte heilige Stätte sei der Garizim ", und dass sie somit Anlass für die postulierten "proto-massoretischen " Korrekturen gaben. ${ }^{51}$ An anderer Stelle führt er aus, dass "die beiden hier besprochenen unkorrigierten Lesarten des Samaritanus und der ursprünglichen Septuaginta [Dtn 12; 27,4; D. J.] auf eine diskrete, aber unüberhörbare Erwähnung des Garizim-Heiligtums im ältesten erreichbaren Deuteronomiumstext « hinweisen. Dies stärke »die Auffassung, das Deuteronomium sei ursprünglich in Efraim-Israel beheimatet $\ll .52$

\section{Folgerungen}

Die zuletzt referierten Überlegungen Schenkers lassen Zweifel aufkommen, inwieweit die Frage nach der »Ursprünglichkeit « der einen oder anderen Lesart in den oben besprochenen Texten sachgemäß ist. Gegen das Verfahren, textkritische Einzelfallentscheidungen zu treffen, ist einzuwenden, dass Aporien bei der Beurteilung inhaltlich verwandter Texte unvermeidbar scheinen. Im Fall von Dtn 27,4 betrifft dies etwa die Not-

47 Schenker, Textgeschichtliches, 107.

48 Schenker, Textgeschichtliches, 108-113. Kartveit, Origin, 228-257.300-309, dagegen verweist darauf, dass die kontrahierte Form nicht zwangsläufig samaritanische Verfasserschaft anzeigt, weil auch römische und christliche Autoren die Wendung Argarizim o. ä. verwenden.

49 Schenker, Textgeschichtliches, 115, Hervorhebung dort.

50 Schenker, Textgeschichtliches, 116.

51 Schenker, Textgeschichtliches, 116.

52 Schenker, Textgeschichtliches, 118. 
wendigkeit, Jos 8,30-35 extrem spät zu datieren. ${ }^{53}$ Zudem spielen inhaltliche Kriterien bei den Optionen für die eine oder andere Lesart in Dtn 27,4 eine große Rolle. Da die Frage nach einer jeweils »ursprünglichen « Lesart nicht befriedigend zu klären ist und da es sich bei den aufgezeigten Alternativlesarten in Dtn 11,30, Dtn 12 und Dtn 27,4 nicht um unbeabsichtigte Schreibfehler, sondern um zielgerichtete Varianten handelt, erscheint es mir im vorliegenden Fall angemessener, die samaritanische Tradition insgesamt und daneben die masoretische Fassung als zwei Textüberlieferungen zu beschreiben, die zumindest zeitweise nebeneinander tradiert und verteidigt wurden. ${ }^{54}$ Unter religionsgeschichtlichen Gesichtspunkten verweisen sie auf die produktiven Auseinandersetzungen zwischen den Kultgemeinden auf dem Garizim und in Jerusalem ab der spätpersischen Zeit.

Für die samaritanische Tradition ist der Berg Garizim ein durch die topographische Inszenierung in Dtn 11 und Dtn 27 herausgehobener Kultort und entspricht damit dem in Dtn 12 umschriebenen Platz der legitimen Jhwh-Verehrung. Die eher vorsichtigen Einschätzungen in dieser Frage etwa von Veijola, Nihan und Schenker beruhen darauf, dass sie nicht die samaritanische Textform als Ganze betrachten, sondern sich textkritische Entscheidungen im Einzelfall vorbehalten bzw. diese etwa hinsichtlich Dtn 12 (Nihan) oder Dtn 11,30 (Schenker) offen lassen. Selbst wenn die samaritanische und die masoretische Textüberlieferung nicht als zwei nebeneinander bestehende Textformen angesehen werden und textkritische Einzelfallentscheidungen zugunsten der »Ursprünglichkeit " des Smr in Dtn 27,4 und Dtn 12 getroffen werden, kann dies schwerlich allein im Sinne einer Konzession gegenüber Ansprüchen der Gemeinde auf dem Garizim verstanden werden. Vielmehr legt sich auch in diesen Fällen eine Deutung des Berges Garizim als dem in Dtn 12 umschriebenen Platz legitimer Jhwh-Verehrung nahe. Unter den genannten Voraussetzungen drängt sich die Folgerung auf, dass das Deuteronomium zumindest teilweise in Samaria entstand. ${ }^{55}$ Auch weitergehende diesbezügliche Überlegungen zur Entstehung des Pentateuch bzw. des Hexateuch sind nicht völlig von der Hand zu weisen. ${ }^{56}$ Solche großflächigen Folgerungen lassen sich jedoch angesichts der schmalen Textbasis kaum nachvollziehbar begründen. Auch von daher halte ich die hier unterbreitete Annahme für angemessener, dass die Unterschiede zwischen den Lesungen von Smr und M in Dtn 11, Dtn 12 und Dtn 27 auf die produktiven Auseinandersetzungen der beiden Jhwh-Kultgemeinden in Jerusa-

\footnotetext{
53 S. o. Anm. 40.

54 Kartveit, Origin, 305; Geiger, Gottesräume, 253.

55 Schenker, Textgeschichtliches, 118.

56 Hjelm, Mt. Gerizim, 35.
} 
lem und auf dem Garizim in spätpersischer und hellenistischer Zeit hinweisen, ohne dass einer der Lesarten der »Vorzug « im Sinne einer »Ursprünglichkeit « zu geben ist.

Komplexer als die Beurteilung der samaritanischen Textüberlieferung gestaltet sich die Einschätzung der masoretischen Tradition. So zieht etwa Tigay auch bei der Annahme von "Ebal « als ursprünglicher Lesart in Dtn 27,4 die Verbindungen zu Dtn 12 aus. Er hält es für möglich, dass "the Deuteronomic movement originally intended Mount Ebal to become sthe place that the Lord will chose to establish His name « .57 Die These, dass der Ebal im Zentrum der Jhwh-Verehrung stand, wird von Sandra L. Richter ausgebaut. ${ }^{58}$ Sie hält die »Ebal tradition « für eine alte Überlieferung aus vorköniglicher Zeit und dementsprechend die Lesart »Garizim « in Dtn 27,4 für eine späte samaritanische Korrektur. Analog zu Praktiken in altorientalischen Kulturen sei die Aufstellung eines mit dem Jhwh-Namen beschriebenen Monuments intendiert, wobei Dtn 11,29-32 und Dtn 27,1-8 darüber informierten, »how, when, and where Yahweh's inscribed monument is to be installed ".59 Richter verweist auf die archäologischen Funde am Ebal und folgert: »Thus, archaeology confirms that something like the report of Deut. xxvii 1-8 could have happened here «. ${ }^{60}$ Die Einwände gegen Richters Thesen liegen auf der Hand: (a) Vom Aufstellen einer oder mehrerer Stelen mit dem JhwhNamen ist weder in Dtn 11 noch in Dtn 27 die Rede. ${ }^{61}$ (b) Dtn 27 bietet keinen "report ", sondern Anweisungen Moses, die erst nach der Landnahme ausgeführt werden sollen. (c) Richter stützt sich auf eine zumindest umstrittene Interpretation der archäologischen Funde am Ebal.62 Auf jeden Fall wurden dort keine aufgerichteten Steine oder ähnliche Monumente entdeckt. Die zentrale Stellung des Ebal im masoretischen Text kann demnach nicht darauf zurückgeführt werden, dass Dtn 11 und Dtn 27 eine alte Kulttradition aufnehmen. Eine Erklärung für das inhaltliche Profil der masoretischen Tradition in den hier besprochenen Überlieferungen zum Garizim sollte vielmehr literarisch-topographischen Gesichtspunkten folgen und im weiteren Textumfeld von Dtn 11,29-31 und Dtn 27,1-13 gesucht werden.

57 Tigay, Deuteronomy, 487.

58 Richter, Place.

59 Richter, Place, 348.

60 Richter, Place, 364.

61 Richter, Place, 344 f., versucht die Verbindung über die in Dtn 12 u. ö. zu findende Wendung, dass Jhwh seinen Namen an einen Ort setzen will, herzustellen. Sie verweist auf akkadisch šuma šakānu »seinen Namen setzen " und erklärt, im altorientalischen Kontext meine eine solche Formulierung die Aufstellung eines beschrifteten Monuments.

62 S. o. Anm. 17. 
So müsste die Reihe von zwölf Flüchen (Dtn 27,14-26) im Sinne des masoretischen Textes von Dtn 27,4 zu verstehen sein. ${ }^{63}$ Da die Flüche von Leviten gesprochen werden, die nach Dtn 27,12 auf dem Garizim stehen, wird auch der Garizim implizit zum Ort des Fluchs. Die masoretische Tradition zielt demnach in erster Linie darauf, den Eindruck zu vermeiden, der Garizim könne der in Dtn 12 umschriebene legitime Kultplatz sein. Demgemäß beschreiben Jos 8,30-35 die in Dtn 11 und Dtn 27 gegebenen Weisungen im Sinne eines vorläufigen oder situativen Kults. ${ }^{64}$ Erst nach der Überführung der Lade und dem Kauf eines Bauplatzes für das Heiligtum (II Sam 24) wird Jerusalem bzw. der dortige Tempel als der Platz angezeigt, an den Jhwh seinen Namen setzt (I Reg 8).65

In diesem Verstehenszusammenhang hat die literarische Inszenierung der Toponyme Sinai und Horeb im Deuteronomium einen wichtigen Platz. ${ }^{66}$ In Dtn 33,2 bezeichnet Sinai eine nicht weiter differenzierte Region. ${ }^{67} \mathrm{Jhwhs}$ irdischer Wohnort ist kein Berg. Somit muss auch der Platz, an dem er verehrt wird, kein Berg sein. Das im Exodusbuch mehrfach »Berg Sinai" genannte Toponym (Ex 19,11.20.23; 24,16 u. ö.) wird im Deuteronomium nur mehr als namenloser »Berg « in der »Einöde « (»Horeb «) vorgestellt (u.a. Dtn 1,6; mehrfach in Dtn 9 f.). ${ }^{68}$ Mit der »Regionalisierung « des Sinai und der Verlegung des »Berges « in eine nicht näher lokalisierbare »Einöde « erreicht das Deuteronomium, dass jeder Zusammenhang zwischen der Präsenz Jhwhs und dem von den Samaritanern kultisch genutzten »Berg Garizim « auszuschließen ist. Dieser Aspekt gilt auch für die Beschreibung der natürlichen Lage: der Garizim liegt weder im fernen Süden bei Seïr oder Paran wie der Sinai (Dtn 33,2) noch in der »Einöde ", sondern im Kulturland. Daran lassen weder Dtn 11,29f. noch

63 Dtn 27,14-26 erscheinen manchen Auslegenden im Erzählzusammenhang nicht einsichtig, da der Stamm Levi auf dem Garizim stehen soll, dem Berg des Segens (V. 12). Daher werden die Verse mitunter als Sonderüberlieferung interpretiert: von Rad, Deuteronomium, 118; Nielsen, Deuteronomium, 246f.; Rüterswörden, Deuteronomium, 171.

64 Vgl. Geiger, Gottesräume, 253.

65 Die intertextuellen Bezüge von Dtn 12 zu I Reg 8 stellt T. C. Römer, Cult Centralization in Deuteronomy 12: Between Deuteronomistic History and Pentateuch, in: E. Otto/ R. Achenbach (Hg.), Das Deuteronomium zwischen Pentateuch und Deuteronomistischem Geschichtswerk, FRLANT 206, 2004, 168-180, zusammen. Dagegen macht I. Hjelm, Jerusalem's Rise to Sovereignty. Zion and Gerizim in Competition, JSOT.S 404 = Copenhagen International Seminar 14, 2004, 295, darauf aufmerksam, dass die begriffliche Verbindung von בחר mit Jerusalem erst in der Chronik belegt ist (II Chr 6,6; 7,12.16).

66 Vgl. Geiger, Gottesräume.

67 Perlitt, Sinai und Horeb, 304 = Perlitt, Deuteronomium-Studien, 34.

68 S. o. Anm. 23; zum Namen Horeb vgl. Perlitt, Sinai und Horeb, 318 = Perlitt, Deuteronomium-Studien, 45. 
Dtn 27,4 trotz der aufgezeigten Unmöglichkeit einer adäquaten historisch-topographischen Auslegung irgendeinen Zweifel.

Da die masoretische Textüberlieferung die Frage des legitimen Kultplatzes bis in das 1 . Königebuch offen hält, verzichtet auch die über das Deuteronomium hinausgehende Darstellung der Kultgeschichte Israels auf die Bezeichnung »Berg ", wenn Aspekte des regulären Jhwh-Kults beschrieben werden. So ist im Zusammenhang der Erzählungen von der Einnahme Jerusalems durch David und des Tempelbaus unter Salomo an keiner Stelle von einem »Berg" die Rede. Die »Stadt Davids" wird als »Festung «מצודה, d.h. als künstlich hoch gebaute und befestigte Anlage bezeichnet (II Sam 5,7.9). Berge hingegen sind Kultorte nichtjahwistischer Gottheiten. Auf »dem Berg gegenüber Jerusalem « errichtet Salomo ein Heiligtum für »Kemosch, den Gräuel Moabs « (I Reg 11,7; vgl. II Reg 23,13.16). Auch Samaria mit seinem vorgeblich Baal geweihten Tempel (I Reg 16,32) wird als »Berg « bezeichnet (I Reg 16,24). Erst mit der Einführung des Begriffs "Zion « wird innerhalb der bis in die Königebücher reichenden Geschichtsdarstellung die Vorstellung vom Berg als Wohnund Kultort Jhwhs akzeptiert (II Reg 19,31). Die Zurückhaltung gegenüber der Bergmotivik lässt sich noch in den jüdisch geprägten Schriften der hellenistisch-römischen Zeit wahrnehmen, wenn etwa Jud 5,14 und Sir 48,7 von Ereignissen in »Sinai « erzählen und das Toponym nicht als »Berg « kennzeichnen. Die christliche Tradition dagegen redet, im Rückgriff auf den Sprachgebrauch im Exodusbuch, vom »Berg Sinai « (Act 7,30.38; Gal 4,25f.), vermutlich auch deshalb, weil sie nicht auf den Konflikt zwischen dem Jerusalemer Tempel und der Gemeinde am Garizim Rücksicht nehmen musste.

Mount Gerizim is mentioned in Deuteronomy 11 and 27, at the two connecting points between the central core of the book (Deut 12-26) and the chapters which form its framework. Deuteronomy 11,29-30 combines names of different places where cultic activities are carried out immediately after the entrance into the land of Canaan. Gerizim and Ebal are the places where Israel has to demonstrate obedience to Jhwh after forty years of rebellion in the wilderness. Deuteronomy 27,4 poses a problem of textual criticism. The Masoretic text calls the place of the veneration of Jhwh »Ebal « whereas the Samaritan Pentateuch writes » Gerizim «. I interpret the difference as a result of productive discussions between the two cultic centres in Jerusalem and on Mt. Gerizim in Persian and Hellenistic times.

Le livre du Deutéronome fait mention du mont Garizim dans les chapitres 11 et 27. Deutéronome 11,29-30 combine la mention des noms des lieux où se déroulent des activités cultuelles immédiatement après que le peuple d'Israël ou Abraham soient entrés en Canaan. Garizim et Ébal sont les lieux ou Israël est obligé de démontrer l'obéissance envers Jhwh après les quarante ans de révolte dans le désert. Le problème cardinal de Deutéronome 27 est une question de critique textuelle: en Dtn 27,4, le texte massorétique dit que "Ébal « est le lieu ou Jhwh serait vénéré, alors que le texte samaritain écrit: »Garizim«. La différence pourrait être 
le résultat d'une discussion productive entre les temples de Jérusalem et du mont Garizim aux époques perse et hellénistique.

Der Berg Garizim ist in Dtn 11 und Dtn 27 jeweils an den Nahtstellen zwischen dem Kernbestand des Buchs (Dtn 12-26) und den Rahmenkapiteln erwähnt. Dtn 11,29f. stellt Toponyme zusammen, die auf kultische Anfangshandlungen im Land Kanaan verweisen: Garizim, Ebal, Gilgal, More. Garizim und Ebal markieren dabei die Plätze, an denen Israel einen Nachweis seiner Bundestreue nach den von Auflehnung gegen Jhwh geprägten Jahren der Wüstenwanderung zu erbringen hat. Dtn 27 stellt textkritische Probleme. Der masoretische Text in Dtn 27,4 nennt den Berg Ebal als den Platz der ersten Kulthandlung in Kanaan, der samaritanische Pentateuch liest hier»Garizim «. Die Varianten werden als Niederschlag der Auseinandersetzungen zwischen den Gemeinden in Jerusalem und auf dem Garizim in spätpersischer und hellenistischer Zeit gedeutet. 